\title{
Chemistry and Structure of Graphene Oxide via Direct Imaging
}

\author{
SUPPLEMENTARY INFORMATION
}

Shreya H. Dave

Department of Mechanical Engineering, Massachusetts Institute of Technology sdave@mit.edu

Chuncheng Gong

Department of Materials, University of Oxford

chuncheng.gong@materials.ox.ac.uk

Alex W. Robertson

Department of Materials, University of Oxford

alex.robertson2@materials.ox.ac.uk

Jamie H. Warner

Department of Materials, University of Oxford

jamie.warner@materials.ox.ac.uk

Jeffrey C. Grossman*

Department of Materials Science \& Engineering, Massachusetts Institute of Technology

77 Massachusetts Avenue, Room 13-5049

Cambridge, MA 02139 USA

617-324-3566

jcg@mit.edu

*indicates corresponding author 

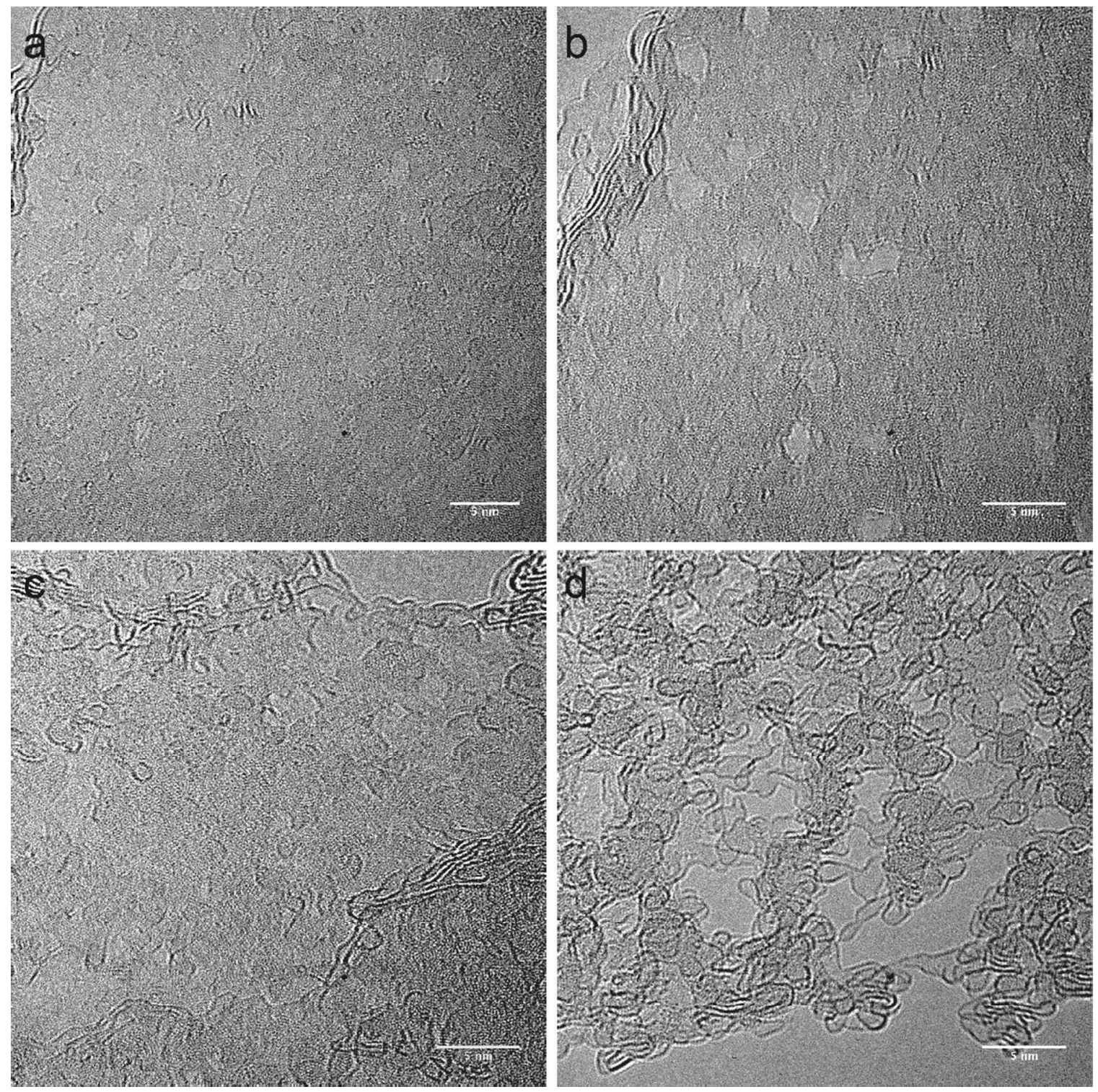

Supplementary Figure 1 (a) and (b) show the same region imaged at room temperature in the ultra-high vacuum of the TEM at $80 \mathrm{kV}$. (b) was captured 3 minutes and 10 seconds after (a), and an a marked increase in hole size and number of holes is apparent. This is an example of the behavior of many different regions of the material under the beam. (c) and (d) show high resolution images of regions at 700C. In (c), the region had not been damaged by the beam at room temperature. In (d), the material had been exposed to the beam at room temperature. The difference in structure and morphology clearly shows the effect of the beam. 


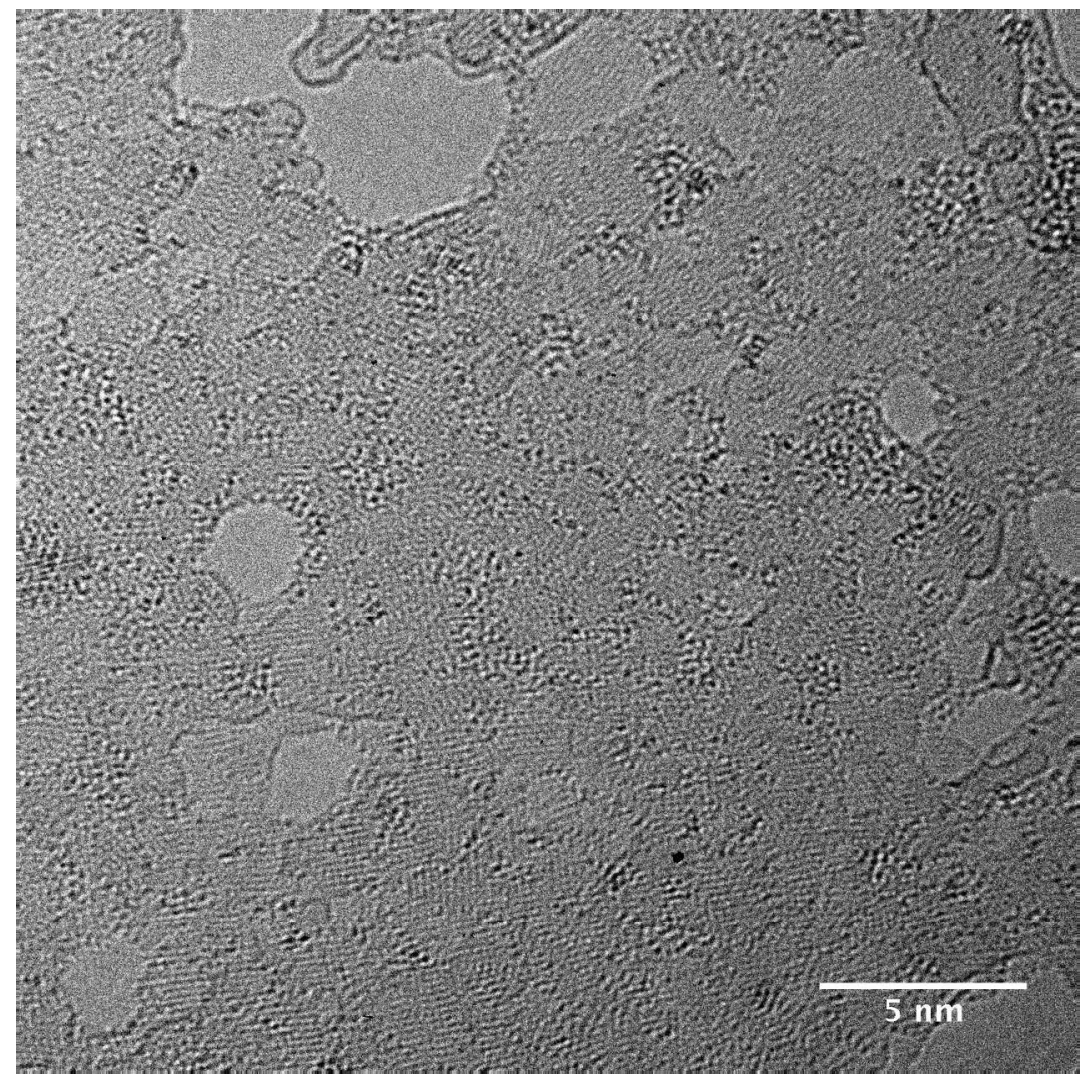

Supplementary Figure 2 Prolonged heating $(700 \mathrm{C})$ and beam exposure causes heavier atoms to migrate into the graphene oxide sample. These are seen as high contrast atoms in the above figure. This region of the sample had previously been damaged by the beam to create the holes that are also visible. The heavier atoms are likely platinum or silicon from the heating coil and silicon nitride TEM chip, respectively. 


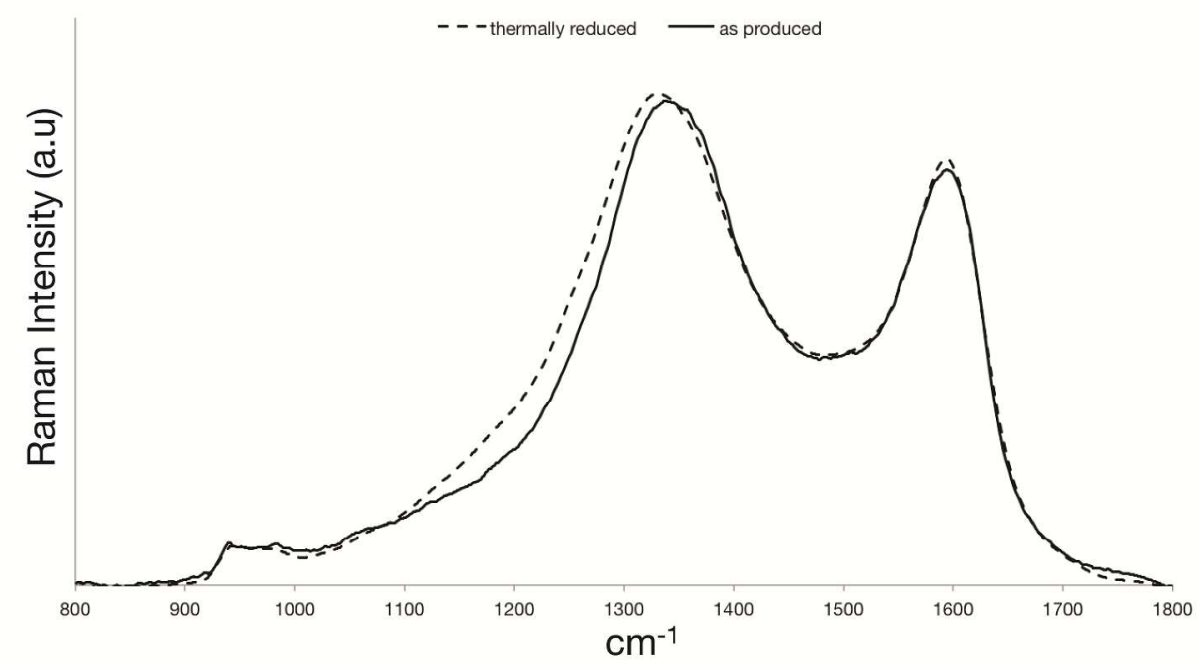

Supplementary Figure 3 Raman Spectroscopy shows a small change in the $I_{D} / I_{G}$ ratio but a general consistency in peak shape. 


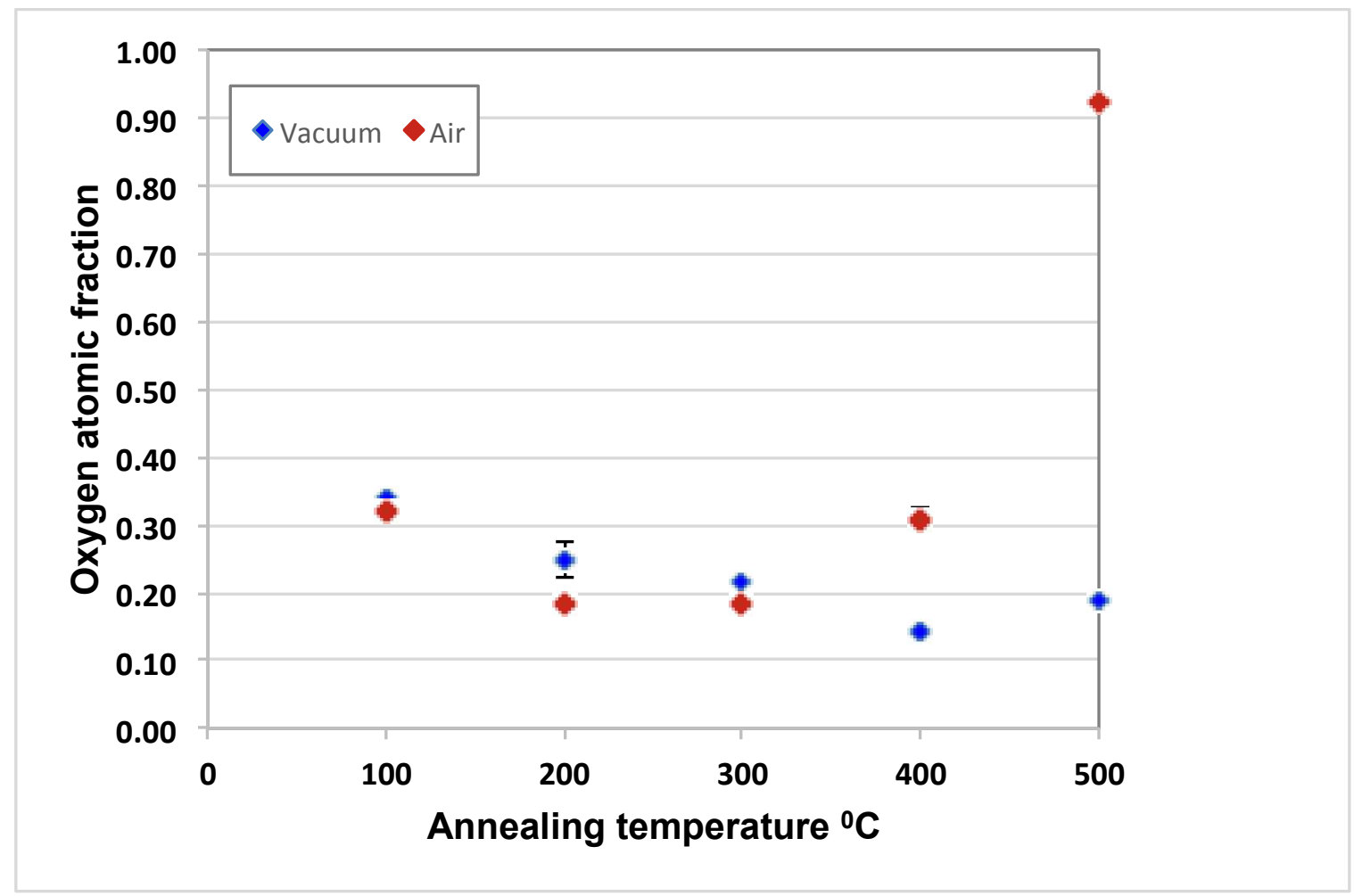

Supplementary Figure 4 XPS data is summarized in terms of the oxygen atomic fraction for samples annealed at temperatures from 100 to $500 \mathrm{C}$. Samples were annealed for 1 hour either in vacuum or in ambient air. Above $400 \mathrm{C}$, the samples annealed in air show high oxygen atomic fraction due to combustion. All data was collected under the vacuum of the XPS at room temperature. Error bars are standard error. 


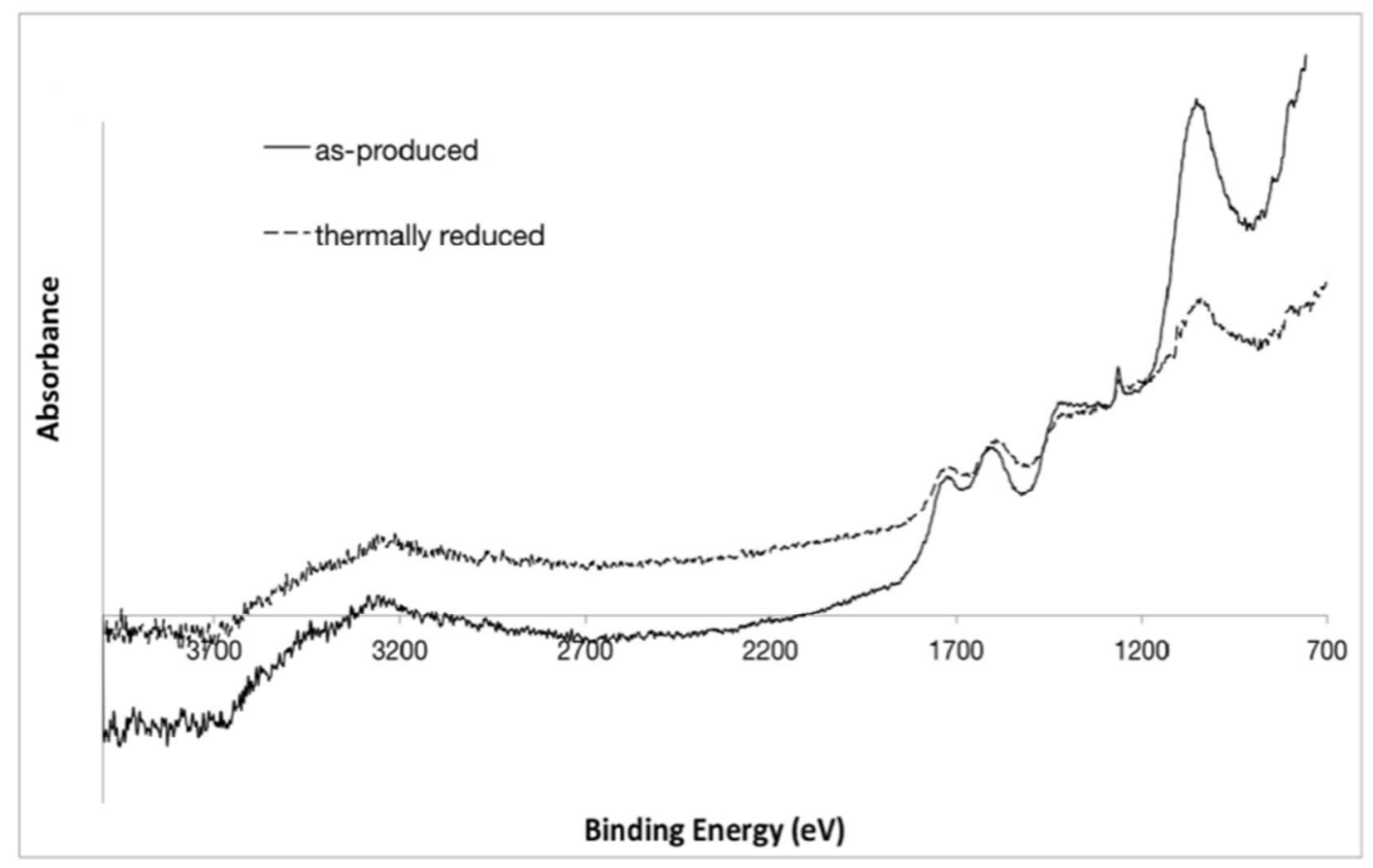

Supplementary Figure 5 FTIR-ATR demonstrates that no drastic chemical change is occurring in terms of surface functional groups. Present in both samples is evidence of the $O-H$ stretch $\left(3300 \mathrm{~cm}^{-1}\right), C=O$ stretch $(1725$ $\left.\mathrm{cm}^{-1}\right)$, water stretch $\left(1600 \mathrm{~cm}^{-1}\right), C-O H$ stretch $\left(1250 \mathrm{~cm}^{-1}\right)$, and $C-O$ stretch $\left(1050 \mathrm{~cm}^{-1}\right)$. The $C-O$ stretch peak at $1050 \mathrm{~cm}^{-1}$ shows a relative reduction in intensity, which is consistent with the XPS results. The broad peak at $3300 \mathrm{~cm}^{-1}$ is likely associated with the surface contaminants and is present in both samples, which are analyzed at room temperature. 


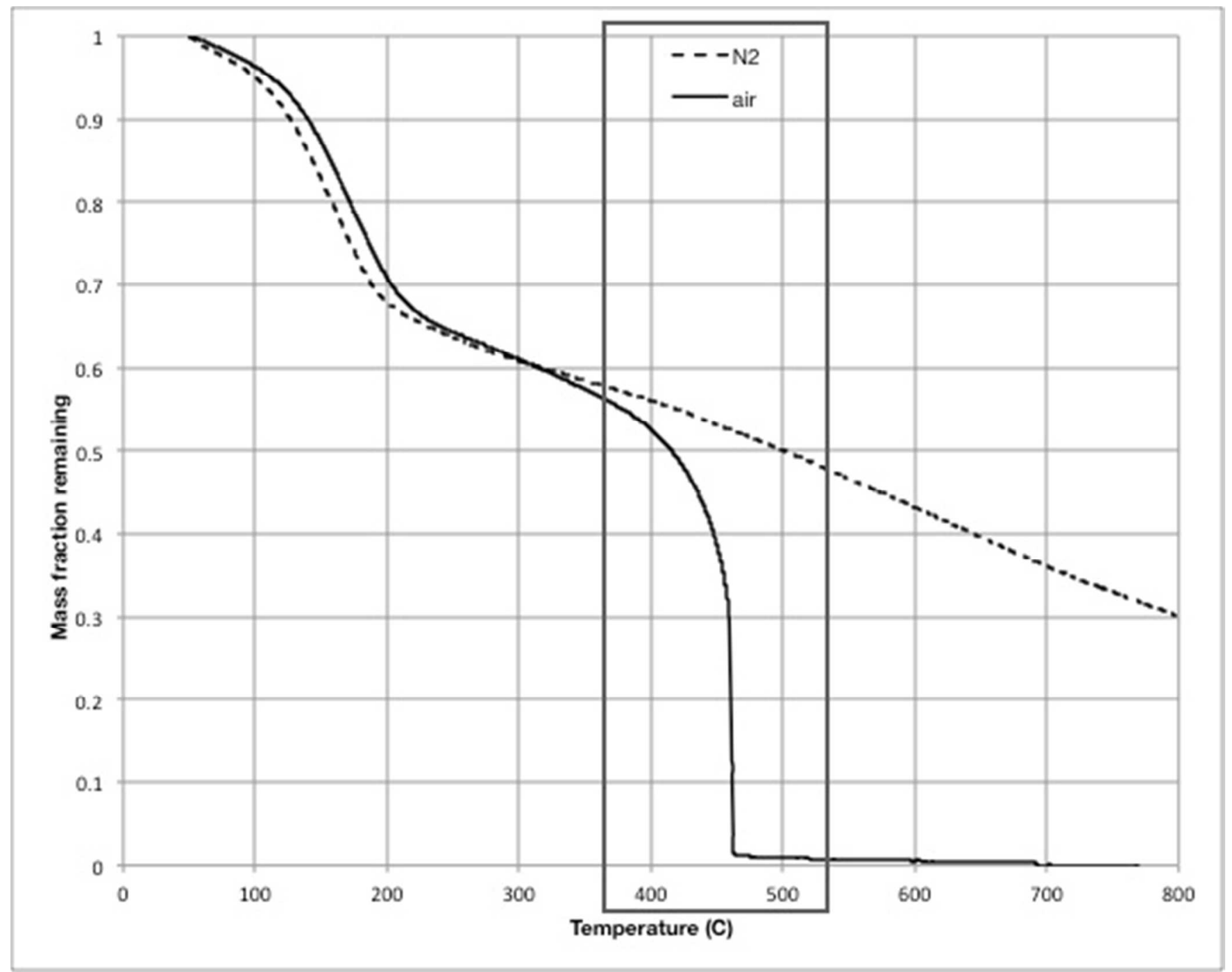

Supplementary Figure 6 TGA data is shown for GO samples heated in both air and nitrogen. GO combusts in air around $420 \mathrm{C}$, causing the big dip in the TGA spectra. A slight change in slope between 400 and $500 \mathrm{C}$ under nitrogen is attributed to the removal of surface contaminants. 

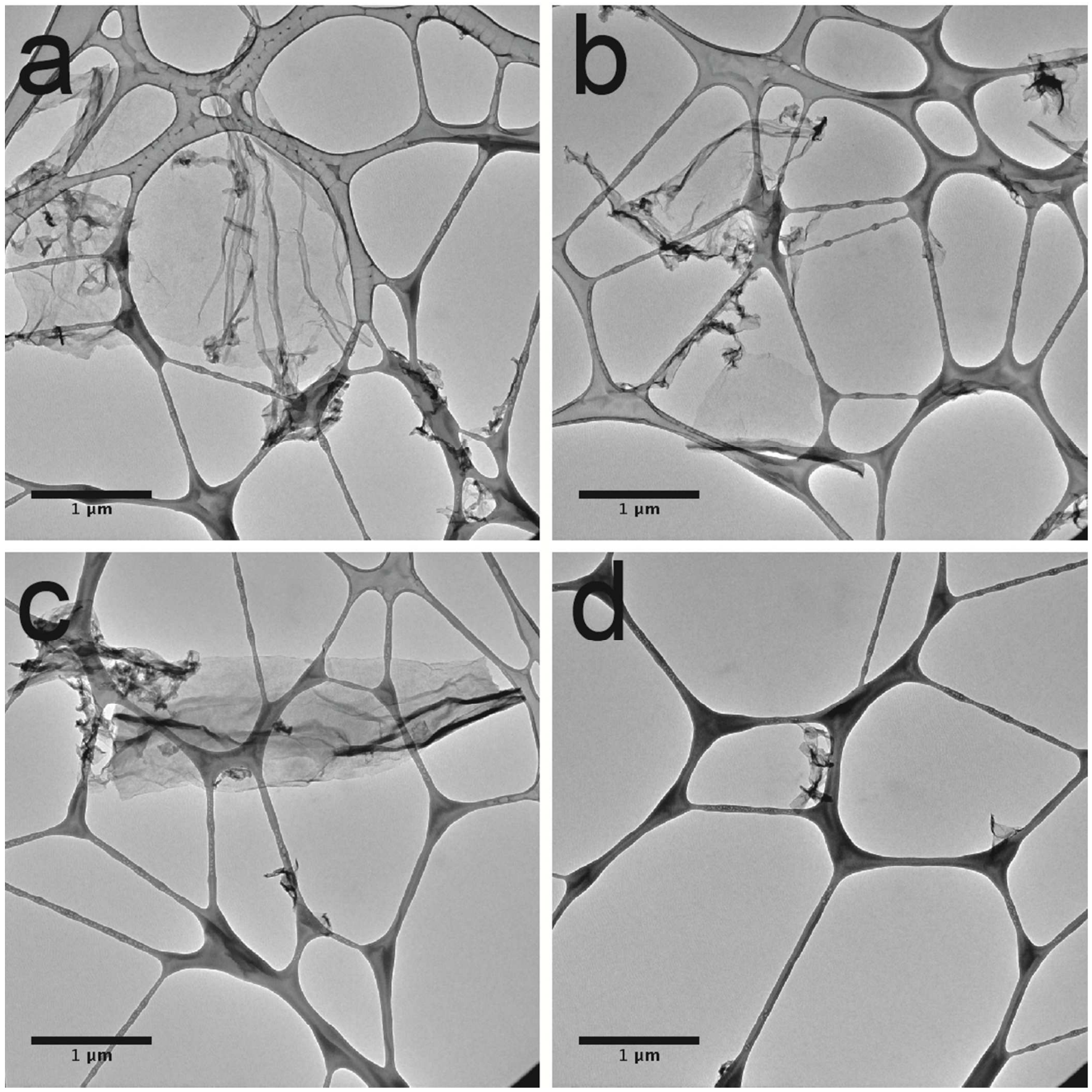

Supplementary Figure 7 Low magnification images of GO deposited on a lacey carbon TEM grid show that the typical flake size is remarkably larger than the $90 \mathrm{~nm}$ reported by the manufacturer. Further, the larger flakes lie flat on the amorphous carbon support, which is more ideal for imaging monolayers and observing contrast. 